\title{
Bunyavirus mRNA synthesis is coupled to translation to prevent premature transcription termination
}

\author{
JOHN N. BARR \\ Institute of Molecular and Cellular Biology, Faculty of Biological Sciences, University of Leeds, Leeds LS2 9JT, United Kingdom
}

\begin{abstract}
Messenger RNA transcription by Bunyaviridae family members is unique within the group of negative-strand RNA viruses as it requires on-going protein synthesis. The long-standing model explaining this phenomenon proposes that the translational requirement is not for a protein product, but instead is for ribosomal translocation along nascent mRNAs. This movement is proposed to disrupt spurious transcription termination signals that otherwise cause premature mRNA truncation leading to a fatal loss of gene expression. This model was tested by introducing translational stop codons into model RNA genomes of Bunyamwera virus, the prototypic member of the Bunyaviridae family. This directly showed that translation of nascent mRNAs prevents transcription termination. While such coupling of transcription and translation is common in prokaryotic systems, these results represent the first report of such obligatory coupling in a eukaryotic cell environment. The results also provide insight into the bunyavirus termination mechanism and suggest it is mechanistically similar to prokaryotic intrinsic termination.
\end{abstract}

Keywords: Bunyamwera; RNA; virus; transcription; termination; translation

\section{INTRODUCTION}

The Bunyaviridae family of segmented RNA viruses comprises Orthobunyavirus, Hantavirus, Phlebovirus, Nairovirus, and Tospovirus genera, which contain many viruses responsible for serious human disease. In recognition of this fact, several bunyaviruses are classified by the National Institutes of Allergy and Infectious Diseases as category A pathogens, responsible for hemorrhagic fevers for which preventative and therapeutic measures are unavailable. The prototypic bunyavirus is Bunyamwera virus (BUNV), which is recognized as a model for investigation of the molecular and cellular biology of pathogens within the Bunyaviridae family.

Bunyaviruses are unique among the group of negativestrand RNA viruses as they require on-going protein synthesis to transcribe functional mRNAs. When protein synthesis is prevented, the products of bunyavirus transcription are prematurely terminated at spurious sites, with little or no synthesis of full-length mRNAs. This phenomenon of obligatorily coupled transcription and translation was first described for Akabane virus and BUNV (Abraham and Pattnaik 1983; Pattnaik and Abraham 1983), but has since been shown to apply to LaCrosse virus (LACV) and

Reprint requestst to: John N. Barr, Institute of Molecular and Cellular Biology, Faculty of Biological Sciences, University of Leeds, Leeds LS2 9JT, UK; e-mail: j.n.barr@leeds.ac.uk; fax: 44-0113-3433167.

Article published online ahead of print. Article and publication date are at http://www.rnajournal.org/cgi/doi/10.1261/rna.436607.
Germiston virus within the same genus (Patterson and Kolakofsky 1984; Vialat and Bouloy 1992). More recently, transcription of Rift Valley Fever virus has been shown to require protein synthesis (Ikegami et al. 2005), suggesting this phenomenon is widespread among bunyaviruses.

Initial attempts to understand the molecular basis for the protein synthesis requirement utilized protein synthesis inhibitors (Raju and Kolakofsky 1987), and also cell-free transcription systems (Bellocq et al. 1987), and these methods showed the requirement was not for generation of a protein product of either viral or cellular origin. This finding was supported by the demonstration that the premature termination phenotype could be reproduced without protein synthesis inhibitors, achieved by saturating a cell-free LACV transcription system with heterologous transcripts to compete for the limited ribosome pool. This showed premature transcription termination did not correlate with reduced protein synthesis, but instead with reduced translation of bunyavirus mRNAs. This was further supported by the subsequent demonstration that the translational requirement could be supplied by movement of $40 \mathrm{~S}$ ribosomal subunits along the bunyavirus transcript (Vialat and Bouloy 1992), which cannot yield a protein product.

To explain these findings, a model was described that proposed the translational requirement of bunyavirus transcription was solely for translocation of ribosomes along nascent viral mRNAs (Fig. 1; Bellocq and Kolakofsky 1987). Central to the model was the proposal that transcription 

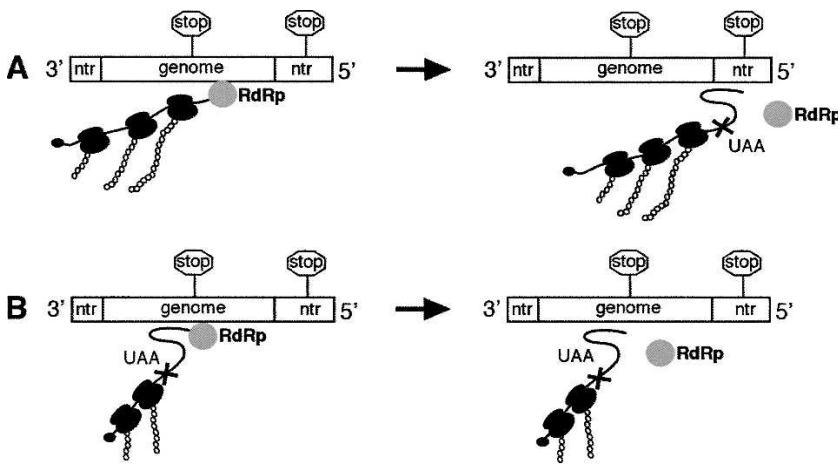

FIGURE 1. Model of obligatorily coupled bunyavirus transcription and translation. (A) Transcription termination requires RNA interactions involving the termination signal (shown as stop signs) or its complement in mRNA. Translating ribosomes prevent formation of interactions, so termination signals within genome coding regions are suppressed. Termination signals within NTRs are active, as these regions are not translated, thus allowing RNA interactions to form (shown as a loop). (B) When translation is inhibited, RNA interactions can form, thus activating previously silent termination signals within coding regions.

termination required formation of an RNA interaction involving the nascent strand. The ability of the ribosome to disrupt termination was due to its ability to disrupt this interaction. Importantly, ribosomes cannot affect termination events that form the authentic mRNA $3^{\prime}$ end, as the termination signal is located within the $5^{\prime}$ NTR (Fig. 1A). However, the segment coding regions contain transcription termination signals that exhibit close similarity to the authentic termination sequence. Therefore, the model proposes that the role of translocating ribosomes is to suppress these spurious signals within the coding region. When ribosome translocation is prevented, these termination sites are active and lead to synthesis of truncated transcripts, and a fatal loss of gene expression (Fig. 1B).

While this model is consistent with available experimental evidence, it has not been tested directly. Here, the first direct test of this model is presented. Reverse genetics was used to engineer translation stop codons into model BUNV segments to unequivocally prevent ribosome translocation along nascent mRNAs. The results show ribosome translocation prevents transcription termination. In addition, the results provide support for a critical role of RNA interactions in the termination mechanism and suggest that bunyavirus termination and prokaryotic intrinsic transcription termination may be mechanistically related.

\section{RESULTS}

\section{Transcription termination within a segment ORF}

As described above, transcription of full-length bunyavirus mRNAs requires on-going protein synthesis, and the current model to explain this phenomenon posits that translocating ribosomes are required to block the activity of transcription termination signals within segment coding regions (Bellocq and Kolakofsky 1987). The central prediction of this model is that the authentic transcription termination signal will not function when located within an actively translated coding region. To test this prediction the $S$ segment termination signal was inserted within the Renilla coding region of previously described plasmid pBUN-S(ren) (Fig. 2B). Resulting plasmid pBUN-T-BsrGI was used to generate model segment BUN-T-BsrGI, which contained identical upstream (T1-UP) and wild-type (T1) transcription termination signals, and their activity was examined by directly visualizing transcribed RNAs using agarose-urea gel electrophoresis (Fig. 2C).

RNA analysis of BUN-T-BsrGI transcription failed to detect synthesis of mRNAs terminated at the T1-UP site.
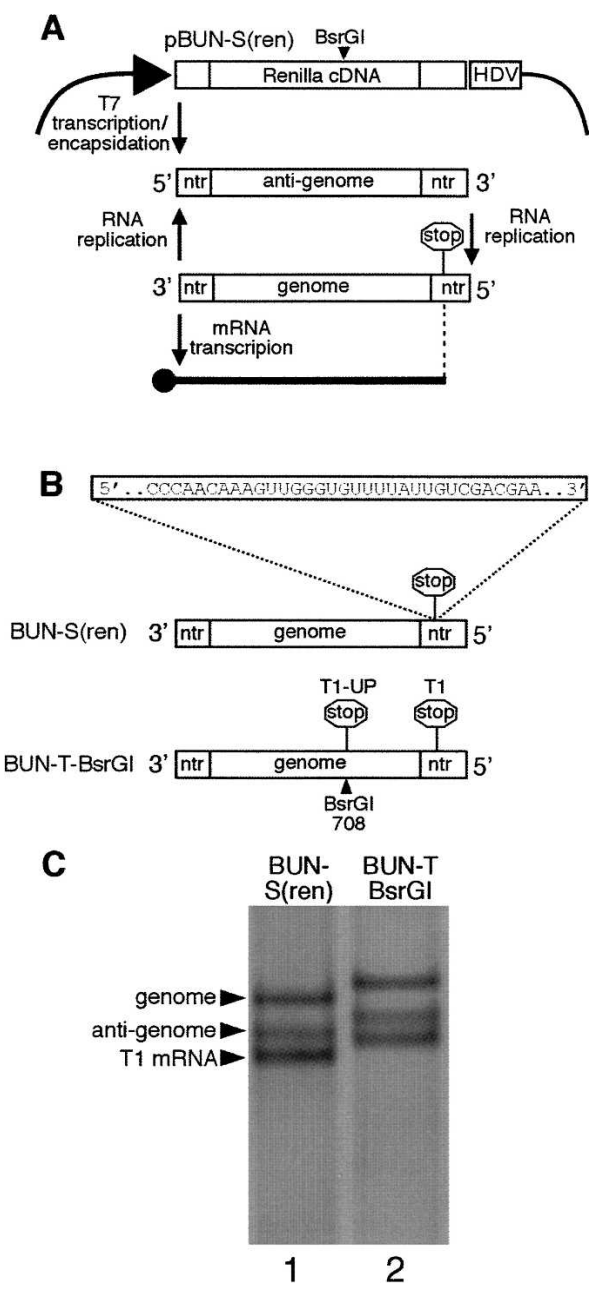

FIGURE 2. The BUNV transcription termination signal is nonfunctional when positioned within an ORF. (A) Schematic of pBUN-S(ren) and the RNAs expressed by the model BUNV segment it encodes. $(B)$ Schematic of model BUNV segment BUN-T-BsrGI, which contains the authentic termination signal (T1) and a duplicate termination signal within the coding region (T1-UP). (C) Direct analysis of RNA synthesized by BUN-S(ren) and BUN-T-BsrGI using agarose-urea gel electrophoresis shows the internal T1-UP signal is inactive. 
Instead, all transcripts terminated at the wild-type $\mathrm{T} 1$ signal located within the $5^{\prime}$ NTR (Fig. 2C, lane 2). The RNA synthesis profile of BUN-T-BsrGI matched that of wildtype BUN-S(ren) (Fig. 2C, lane 1). These results show the inserted T1-UP signal was not active in the Renilla coding region, thus confirming the prediction of the model.

\section{Translocation prevents transcription termination}

The above results showed the T1-UP transcription termination signal was nonfunctional when located within the Renilla ORF. While this observation was consistent with the model, it was also possible that lack of T1-UP activity was not due to translocation alone, but was also affected by the new location of T1-UP and the surrounding sequences.

To more accurately test the role of translocation in preventing termination, a mutagenesis strategy was devised that allowed translocation across the termination signal to be controlled by altering a single nucleotide. This allowed analysis of how ribosome translocation affected T1-UP signaling using minimal template change.

This strategy involved generating a cDNA fragment representing the BUNV termination signal directly preceded by a UAA stop codon from annealed oligonucleotides (Fig. 3A). This fragment was inserted at a BglII site introduced at nucleotide 697 of the Renilla cDNA within pBUN-S(ren), such that the UAA stop codon was in frame with the Renilla ORF (Fig. 3A). Resulting plasmid pBUN-T697 was then modified by inserting either 1 or 2 nucleotides (nt) directly upstream of the insertion site to generate pBUN$\mathrm{T} 697+1$ and pBUN-T697 + 2, respectively (Fig. 3A). These alterations shifted the position of the fragment relative to the Renilla ORF and changed the reading frame in which the UAA codon was located. Therefore, while the stop codon was in frame with the Renilla ORF in plasmid pBUN-T697, it was out of frame in plasmids pBUN-T697(+1) and pBUN-T697(+2).

These three plasmids were then used to generate corresponding BUNV segments BUN-T697, BUN-T697 + 1, and BUN-T697 + 2, as described in Materials and Methods. The model predicted the T1-UP signal of BUN-T697 would be active, as ribosome translocation was blocked. In contrast, the T1-UP signals of BUN-T697 + 1 and BUN-T697 + 2 were predicted to be inactive, as ribosomes would translate their T1-UP blocked upstream. sequences. These predictions were tested by examining the T1-UP activity using agarose-urea gel electrophoresis, as described above. This analysis detected transcription termination at the T1-UP site of BUN-T697 and a corresponding reduction in termination activity at the wild-type $\mathrm{T} 1$ site (Fig. 3B, lane 1). However, termination at the T1-UP signal of either BUN-T697(+1) or BUNT697(+2) was not detected (Fig. 3B, lanes 2,3). Together, these results show that the T1-UP signal is only active when ribosome translocation is prevented, thus fully supporting the proposed model.

To confirm that translational inhibition of termination was a phenomenon occurring throughout the coding region, the UAA/T1-UP oligonucleotide was also inserted at a downstream position 936 within pBUN-S(ren), such that the UAA codon was in frame with the Renilla ORF (Fig. 3C). Resulting plasmid pBUN-T936 was then modified by insertion or deletion of $1 \mathrm{nt}$ to shift the reading frame of the UAA codon, generating pBUN-T936(+1) and pBUNT936(-1), respectively (Fig. 3C). These plasmids were used to generate corresponding model segments, and the activity of their T1-UP sites was examined as described above. This analysis showed the BUN-T936 T1-UP signal was
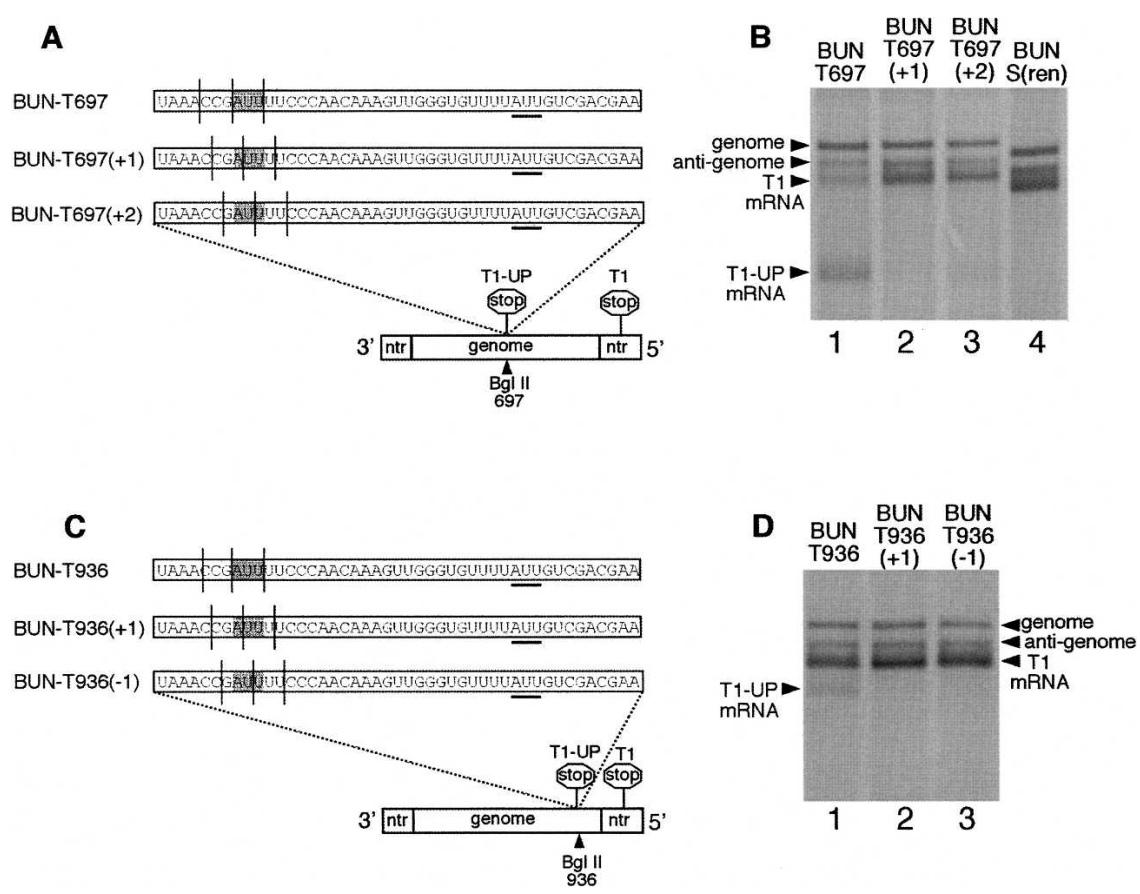

FIGURE 3. Transcription termination at T1-UP only occurs if ribosome translocation is prevented. $(A, C)$ Schematic representation of model BUNV segments BUN-T697 and BUNT936 and their derivatives. The T1-UP signal preceded by a UAA stop codon (shaded) was inserted at nucleotides 697 or 936 of the Renilla cDNA, such that the stop codon was in frame with the Renilla ORF (vertical lines). The two segments were modified by insertion or deletion of single nucleotides to position the UAA stop codon in alternative reading frames. $(B, D)$ Direct analysis of RNAs synthesized by BUN-T697, BUN-T936, and their derivatives using agarose-urea gel electrophoresis. T1-UP signals were only active when translocation was 
active, whereas the T1-UP signals within BUN-T936(+1) and pBUN-T936(-1) were inactive (Fig. 3D, lanes 2,3). These results fully agree with those of the previous section, showing that T1-UP was only active when ribosome translocation was prevented. Together these results show translational inhibition of termination is a general phenomenon that occurs throughout the coding region. Interestingly, T1-UP was more active at position 697 than 936 (Fig. 3, lanes B1,D1, cf. relative abundances of T1-UP and T1 mRNAs). The reason for this difference is unknown.

\section{Termination is affected by stop codon proximity}

The results presented above show that transcription termination signals positioned in an ORF were inactive, but their activity could be restored by positioning an in-frame stop codon directly upstream.

However, these results show that termination activity was only partially restored, as evidenced by detection of transcripts that failed to terminate at the T1-UP site and instead terminated at the downstream T1 signal (Fig. 3B,D, lane 1). In contrast, we previously showed the wild-type T1 signal was extremely efficient at signaling termination, with read-through transcription products being undetectable (Barr et al. 2006). This discrepancy between termination signaling of T1 and T1-UP was unexpected, as both signals were identical and similarly located downstream of stop codons.

To explain this difference, we hypothesized that reduced T1-UP activity was due to the proximity of the upstream stop codon, located just 2 nt away (Fig. 3A,C), and that a ribosome terminating translation at this location may disrupt transcription termination without translocating across the termination sequence. Consistent with this hypothesis, the $\mathrm{T} 1$ signal in BUN-S(ren) is located over $50 \mathrm{nt}$ downstream from the Renilla stop codon. This hypothesis predicted that if the distance between the stop codon and the T1-UP signal was increased to at least $50 \mathrm{nt}$, then it would exhibit a signaling ability equal to $\mathrm{T} 1$.

To test this prediction, model segment BUN-T697 was modified by incorporating in-frame UAA stop codons $121 \mathrm{nt}$ upstream of the T1-UP signal (Fig. 4A). The RNA synthesis activity of resulting template BUN-TSTOP was then analyzed using agaroseurea gel electrophoresis to determine the signaling ability of the T1-UP site. This analysis showed exclusive transcription termination at T1-UP, with no detectable termination at the down-

stream T1 signal, showing that stop codon proximity profoundly affects termination signaling ability.

\section{DISCUSSION}

The model describing the dependence of bunyavirus transcription on protein synthesis posits that ribosomes must translate viral mRNAs to block the activity of transcription termination signals within coding regions (Fig. 1). The model also suggests that ribosomes block transcription termination by disrupting RNA interactions that are critical for termination signaling. In this report, the affect of ribosome translocation across the BUNV termination signal was directly examined. The results show that translocation abrogates transcription termination, thus confirming the central tenet of the model. Consequently, these results support the proposed role of RNA interactions in the termination mechanism, as the ability of ribosomes to disrupt RNA structures is well documented (Wright and Hayward 1987).

To gain understanding of the termination mechanism, my laboratory is interested in locating these proposed RNA interactions. Although the current study cannot unequivocally determine their location, they likely occur within the termination sequence itself. The evidence is that the site of transcription termination in this and previous studies (Barr et al. 2006) always corresponds to the position of the inserted termination signal, suggesting all essential signaling elements are contained within the inserted sequence. Inspection of the BUNV termination signal to identify potentially interacting nucleotides reveals a strong G/C-rich hairpin that is conserved in many members of the Orthobunyavirus genera and is known to contribute toward termination signaling ability (Barr et al. 2006).

The results show that termination signaling ability was affected by not only the presence of an in-frame stop codon, but also by how far upstream of the termination signal this codon was located. When it was located $121 \mathrm{nt}$

A

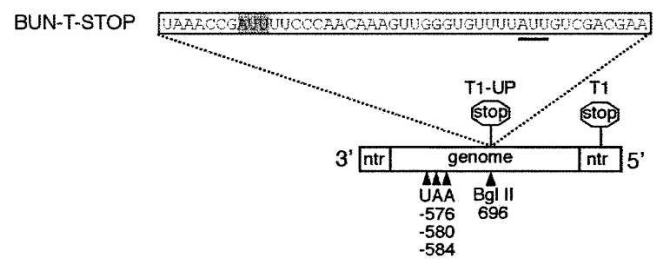

FIGURE 4. T1-UP termination ability is influenced by position of the upstream stop codon. (A) Model BUNV segment BUN-T697 was modified by inserting a stop codon 121 nt upstream to generate BUN-T-STOP. (B) Direct analysis of RNAs synthesized by BUN-T697 and BUN-TSTOP using agarose-urea gel electrophoresis showed T1-UP activity was increased when ribosome translocation was blocked upstream. 
upstream, termination signaling was fully active, but when located just $2 \mathrm{nt}$ upstream, termination signaling was reduced. In the context of the model, we propose that when the translocating ribosome reaches the stop codon 2 nt upstream of T1-UP, its physical size allows it to partially disrupt the crucial RNA interactions, even though the ribosome does not directly translocate across the corresponding sequences. Conversely, ribosomes that reach the stop codon $121 \mathrm{nt}$ upstream cannot affect these RNA interactions, as they occur too far downstream.

Interestingly, the BUNV termination sequence itself contains an internal stop codon (Figs. 3A,C, 4A; AUU underlined), and for templates BUN-T697 + 2 and BUNT936 - 1, this is the only in-frame stop codon upstream of the termination signal. The results showed the T1-UP signals within these templates were inactive, and in the context of the model, we suggest that ribosome translocation up to the internal stop codon disrupts the critical RNA interaction, leading to a loss of termination activity.

The dependence of bunyavirus transcription on translation, and the apparent requirement of RNA interactions, is a unique characteristic within the group of negativestrand RNA viruses. This may reflect fundamental differences between transcription termination mechanisms of viruses within this group, and this suggestion is supported by the identification of the BUNV termination signal, which showed no sequence similarity to other negativestrand RNA virus termination signals (Barr et al. 2006).

To the best of my knowledge, no other transcription mechanism occurring within the eukaryotic cell exhibits an obligatory dependence on cotranslation of the nascent mRNA. However, such coupling between transcription and translation is well documented in prokaryotic systems (Roland et al. 1988). The example of rho-independent (intrinsic) termination is particularly relevant to the current study, as this process is also disrupted by translation. Intrinsic termination occurs when the polymerase pauses and a hairpin forms in the nascent mRNA (Gusarov and Nudler 1999; Yarnell and Roberts 1999). Hairpin formation draws the nascent strand out of the polymerase by virtue of a weak U/A hybrid within the active site and leads to release of the transcript. Ribosomes are proposed to disrupt intrinsic termination by preventing formation of this hairpin.

BUNV transcription termination bears a close resemblance to this mechanism in two critical ways. First, as the current study shows, translation disrupts termination signaling. Second, RNA interactions are likely involved in the termination process. It is also interesting to note that the two critical cis-acting sequence elements of the intrinsic terminator are also present within the BUNV termination signal, namely, a strong hairpin and also a U/A-rich sequence. These two sequence elements both contribute toward termination signaling, and their possible functional significance is further supported by the observation that they are conserved within the Orthobunyavirus genera.

\section{MATERIALS AND METHODS}

\section{Plasmid constructions}

Previously described plasmid pBUN-S(ren) contains a cDNA encoding a model BUNV segment comprising $S$ segment $3^{\prime}$ and $5^{\prime}$ NTRs flanking the Renilla luciferase coding region (Barr et al. 2003). Transcription of this sequence is controlled by the T7 RNA polymerase promoter and is flanked at the $3^{\prime}$ end by the Hepatitis delta virus ribozyme (Fig. 2A). Following transcription, the resulting RNA contains an authentic S segment $3^{\prime}$ terminus, and two additional $G$ residues that are removed following BUNVdirected RNA replication.

Plasmid pBUN-T-BsrGI was derived from pBUN-S(ren) by inserting annealed oligonucleotides comprising the 33-nt-long BUNV S segment transcription termination signal at the naturally occurring BsrGI site at nucleotides 708-713 within the Renilla cDNA sequence (Fig. 2B; Lorenz et al. 1991).

Plasmid pBUN-T697 was generated in two stages. First, pBUN$\mathrm{S}$ (ren) was modified by engineering a BglII restriction site at nucleotides 697-702 of the Renilla cDNA using QuikChange mutagenesis (Stratagene). Second, the BglII site was used to insert annealed oligonucleotides comprising the 33-nt-long BUNV S segment termination signal preceded by a translation stop codon (Fig. 3A). The reading frame of the stop codon was changed by inserting 1 or 2 nt directly upstream of the BglII site, again using QuikChange. This strategy was also used to generate plasmid pBUN-T936, in which the annealed oligonucleotide was inserted at a BgIII site introduced at nucleotides 936-941 (Fig. 3C).

Plasmid pBUN-T-STOP was derived from $\mathrm{pBUN}-\mathrm{S}$ (ren) by altering nucleotides 576, 578, 580, 581, 582, and 584 using QuikChange to introduce a UAA stop codon within all three Renilla reading frames at nucleotides 576, 580, and 584 (Fig. 4A). All alterations were checked by sequence analysis.

\section{Transfections}

Model BUNV RNA segments were generated in BHK-21 cells as described previously (Barr et al. 2003). Briefly, cDNAs expressing a BUNV segment and BUNV S and L ORFs were transfected into BHK-21 cells previously infected with recombinant Vaccinia virus vTF7-3, which expresses T7 RNA polymerase. These expressed components assembled into active templates for BUNV RNA synthesis. Resulting BUNV RNAs were metabolically labeled $14 \mathrm{~h}$ post-transfection using ${ }^{3} \mathrm{H}$ uridine $(33 \mu \mathrm{Ci} / \mathrm{mL}$; PerkinElmer $)$ in the presence of actinomycin-D (10 $\mu \mathrm{g} / \mathrm{mL}$; Sigma) for a total of $6 \mathrm{~h}$, after which total RNA was harvested using RNeasy (Qiagen).

\section{RNA analysis}

Metabolically labeled RNAs were analyzed using agarose-urea gel electrophoresis (Lehrach et al. 1977), which resolves RNAs based on both size and sequence. RNAs were visualized by fluorography and autoradiography.

\section{ACKNOWLEDGMENTS}

I thank Summer Galloway and Tara Wertz-Cartee for technical assistance, and R.M. Elliott (St. Andrews, Scotland) for continued use of cDNAs expressing BUNV S and L ORFs and BUN-S(ren). 
This work was supported by a RCUK academic fellowship, and also in part by an unrestricted research award to the laboratory of Gail W. Wertz from Bristol-Myers Squibb and NIH grant AI12464.

Received December 15, 2006; accepted February 26, 2007.

\section{REFERENCES}

Abraham, G. and Pattnaik, A.K. 1983. Early RNA synthesis in Bunyamwera virus-infected cells. J. Gen. Virol. 64: 1277-1290.

Barr, J.N., Elliott, R.M., Dunn, E.F., and Wertz, G.W. 2003. Segmentspecific terminal sequences of Bunyamwera bunyavirus regulate genome replication. Virology 311: 326-338.

Barr, J.N., Rodgers, J.W., and Wertz, G.W. 2006. Identification of the Bunyamwera bunyavirus transcription termination signal. J. Gen. Virol. 87: 189-198.

Bellocq, C. and Kolakofsky, D. 1987. Translational requirement for La Crosse virus S-mRNA synthesis: A possible mechanism. J. Virol. 61: 3960-3967.

Bellocq, C., Raju, R., Patterson, J., and Kolakofsky, D. 1987. Translational requirement of $\mathrm{La}$ Crosse virus S-mRNA synthesis: In vitro studies. J. Virol. 61: 87-95.

Gusarov, I. and Nudler, E. 1999. The mechanism of intrinsic transcription termination. Mol. Cell 3: 495-504.

Ikegami, T., Won, S., Peters, C.J., and Makino, S. 2005. Rift Valley fever virus NSs mRNA is transcribed from an incoming anti-viralsense S RNA segment. J. Virol. 79: 12106-12111.
Lehrach, H., Diamond, D., Wozney, J.M., and Boedtker, H. 1977. RNA molecular weight determinations by gel electrophoresis under denaturing conditions, a critical reexamination. Biochemistry 16: 4743-4751.

Lorenz, W.W., McCann, R.O., Longiaru, M., and Cormier, M.J. 1991. Isolation and expression of a cDNA encoding Renilla reniformis luciferase. Proc. Natl. Acad. Sci. 88: 4438-4442.

Patterson, J.L. and Kolakofsky, D. 1984. Characterization of La Crosse virus small-genome transcripts. J. Virol. 49: 680-685.

Pattnaik, A.K. and Abraham, G. 1983. Identification of four complementary RNA species in Akabane virus-infected cells. J. Virol. 47: $452-462$.

Raju, R. and Kolakofsky, D. 1987. Translational requirement of La Crosse virus S-mRNA synthesis: In vivo studies. J. Virol. 61: 96-103.

Roland, K.L., Liu, C.G., and Turnbough Jr., C.L. 1988. Role of the ribosome in suppressing transcriptional termination at the pyrBI attenuator of Escherichia coli K-12. Proc. Natl. Acad. Sci. 85: 7149-7153.

Vialat, P. and Bouloy, M. 1992. Germiston virus transcriptase requires active $40 \mathrm{~S}$ ribosomal subunits and utilizes capped cellular RNAs. J. Virol. 66: 685-693.

Wright, J.J. and Hayward, R.S. 1987. Transcriptional termination at a fully rho-independent site in Escherichia coli is prevented by uninterrupted translation of the nascent RNA. EMBO J. 6: $1115-1119$.

Yarnell, W.S. and Roberts, J.W. 1999. Mechanism of intrinsic transcription termination and antitermination. Science 284: $611-615$. 

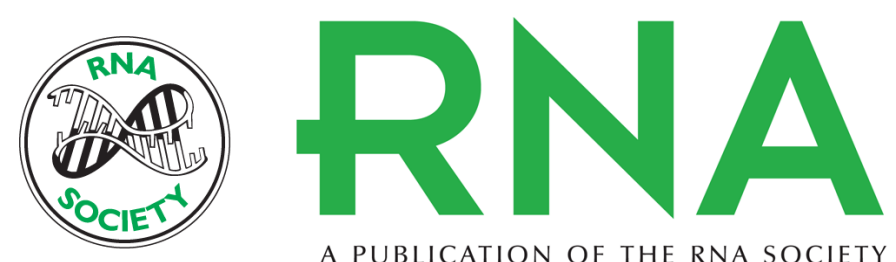

A PUBLICATION OF THE RNA SOCIETY

\section{Bunyavirus mRNA synthesis is coupled to translation to prevent premature transcription termination}

John N. Barr

RNA 2007 13: 731-736 originally published online March 30, 2007

Access the most recent version at doi:10.1261/rna.436607

$\begin{array}{ll}\text { References } & \begin{array}{l}\text { This article cites } 16 \text { articles, } 10 \text { of which can be accessed free at: } \\ \text { http://rnajournal.cshlp.org/content/13/5/731.full.html\#ref-list-1 }\end{array}\end{array}$

License

Email Alerting Receive free email alerts when new articles cite this article - sign up in the box at the Service top right corner of the article or click here. 